\title{
Effect of Plasmoids on Nonlinear Evolution of Double Tearing Modes
}

\author{
J. Ma1,2,a), W. Guo ${ }^{1,2, a)}$, Z. $Y u^{1,2}$, Q. $Y u^{3}$
}

${ }^{1}$ Institute of Plasma Physics, Chinese Academy of sciences, Hefei 230031, China

${ }^{2}$ Center for Magnetic Fusion Theory, Chinese Academy of Sciences, Hefei 230031, China

${ }^{3}$ Max-Planck-Institut für Plasmaphysik, 85748 Garching, Germany

\begin{abstract}
The nonlinear evolution of double tearing mode is numerically investigated in 2D geometry. Long and thin current sheets are found to be formed and become tearing unstable in high Lundquist number regime during the fast reconnection phase, leading to the onset of the secondary and tertiary islands (plasmoids). Eventually the system saturates at a quasi-stationary state with those islands coexisting in two pairs. Interestingly, a new evolution process, characterized by two fast reconnection phases, is also discovered for an intermediate distance between the two resonant surfaces due to the evolution of current ribbons associated with the secondary island. These results are obtained only when the symmetry of magnetic configuration is strictly guaranteed in numerical calculations.
\end{abstract}

Keywords: plasmoids, double tearing mode, magnetic reconnection, MHD instabilities

\section{Introduction}

Magnetic reconnection is one of the most important physical phenomena in space and magnetically confined plasmas[1]. It changes magnetic configuration and releases magnetic energy into bulk plasma heating and kinetic energy. Amongst many magnetic reconnection events, fast reconnection, e.g. during the sawtooth crashes in tokamak plasmas, is of special interest. Recently, analytical[2] and numerical[3]-[5] efforts have been devoted to investigate the stability of a current sheets at high Lundquist number. The current sheet can be tearing unstable and break up into plasmoids, providing a possible explanation for reconnection events faster than the Sweet-Parker reconnection model.

Multiple current sheets systems are also commonly encountered in space and laboratory plasmas. One example is the configuration with central reversed magnetic shear in tokamak discharges. The non-monotonic q-profile can lead to the formation of the internal transport barrier and a higher bootstrap current fraction than the standard H-mode plasmas[6],[7], being a possible configuration for the steady operation of a fusion reactor. However, for such configuration double tearing mode[8]-[19] (DTM) can be excited, resulting in fast magnetic reconnection and confinement degradation[20]-[23].

a)Author to whom correspondence should be addressed. Electronic mail: junma@ipp.ac.cn, wfguo@ipp.ac.cn 
Therefore, DTM is of great interest to fusion research and has been investigated both analytically and numerically in different geometries. As one of the most important aspects in nonlinear evolution of DTM, the change of magnetic configurations by DTM has been intensively investigated before[24]-[28]. A common knowledge is that, when these two resonant surfaces are close enough, the stagger arranged islands at different rational surfaces can grow bigger and triangularly deform, resulting in intensive current density around island's x-point and subsequent explosive flow driving reconnection. In the following phase the islands on different current sheets switch their radial position, and reconnect with open field lines on the opposite $\mathrm{X}$ points, leading to a final quasi-steady state with almost parallel field lines and small residual poloidal flows. These results are obtained with the Lundquist number being much lower than that in tokamak experiments.

In higher Lundquist number regime, recent numerical studies with resistive MHD model revealed that the physical process can be different[1]. Instead of the intensive density around island's x-point, unstable long and thin current sheets can be formed as a result of islands interaction and break up during the nonlinear evolution of DTM, leading to the secondary islands[29] or even tertiary islands[30][31], showing the diversity and complexity of physics in high Lundquist number regime. However, in these studies the symmetry is not well preserved in calculations due to numerical errors. In this case a small numerical error together with a high Lundquist number will result in a sufficiently large electromagnetic force to generate artificial plasma flows and the loss of momentum conservation in numerical calculations, which leads to nonphysical motion of plasmoids in the nonlinear phase such that plasmoids only transiently exist in the reconnection region. This problem has been overcome by the conservative perturbed resistive MHD model[35],[36], which allows to study the nonlinear growth and saturation of DTM with the symmetry well preserved in numerical simulations. This is especially important in simulating the nonlinear mode growth and saturation with high Lundquist numbers, the regime being more relevant to existing tokamak experiments and where plasmoids form.

In this work, the conservative perturbed resistive MHD model[35],[36] is utilized to systematically investigate the nonlinear evolution of DTM, focusing on the regime with sufficiently high Lundquist number and the formation and evolution of plasmoids. The symmetry is well preserved during our numerical simulations. It is found that the system can saturates at a quasi-stationary state with the secondary and tertiary islands coexisting in two pairs. The effect of the distance between the two resonant surfaces is also studied. For an intermediate distance a new kind of nonlinear evolution of DTM with two fast reconnection phases, caused by the secondary island, is observed.

This paper is organized as follows: in Section 2 the conservative resistive MHD model for DTM simulation is presented. In section 3 , the numerical results are reported. Finally the discussion and summary are given.

\section{Physics model}

The DTM can be described by resistive MHD equations including the mass, momentum, energy conservation and the Ohm's laws[37]:

$$
\begin{array}{r}
\partial_{t} \rho+\nabla \cdot(\rho \vec{V})=0 \\
\partial_{t}(\rho \vec{V})+\nabla \cdot\left(\rho \vec{V} \vec{V}-\vec{B} \vec{B}+\left(p+\frac{1}{2} B^{2}\right) \vec{I}-v \nabla \vec{V}\right)=0(2)
\end{array}
$$




$$
\partial_{t}\left(\frac{p}{\Gamma-1}+\frac{1}{2}\left(\rho V^{2}+B^{2}\right)\right)+\nabla \cdot\left[\left(\frac{\Gamma p}{\Gamma-1}+\frac{1}{2}\left(\rho V^{2}+B^{2}\right)\right) \vec{V}-(\vec{B} \cdot \vec{V}) \vec{B}-v \nabla \frac{V^{2}}{2}+\eta \vec{J} \times \vec{B}\right]=0
$$

$$
\partial_{t} \vec{B}+\nabla \cdot(\vec{V} \vec{B}-\vec{B} \vec{V}-\eta \nabla \vec{B})=0(4)
$$

where $\rho, \vec{V}, p, \overrightarrow{\mathrm{B}}, \vec{J}, v, \eta$ are the plasma density, velocity, pressure, magnetic field, current density, viscosity and resistivity respectively. The magnetic divergence constraint $\nabla \cdot \vec{B}=0$ is also applied. $\vec{V}=u_{x} \vec{e}_{x}+u_{y} \vec{e}_{y}+u_{z} \vec{e}_{z}, \vec{B}=B_{x} \vec{e}_{x}+B_{y} \vec{e}_{y}+B_{z} \vec{e}_{z}, \Gamma=\frac{5}{3}$ is the ratio of specific heat, and the plasma energy density is defined as $\rho e=\frac{p}{\Gamma-1}+\frac{1}{2} \rho V^{2}+\frac{1}{2} B^{2}$. Variables are normalized in the following way: $\rho / \rho_{00} \rightarrow \rho, p /\left(B_{00}^{2} / \mu_{0}\right) \rightarrow p, \quad \vec{V} / V_{A} \rightarrow \vec{V}, \vec{B} / B_{00} \rightarrow \vec{B}, \vec{J} /\left(B_{00} / \mu_{0} a\right) \rightarrow \vec{J}$, $v /\left(a V_{A} \rho_{00}\right) \rightarrow v, \eta /\left(\mu_{0} a^{2} / \tau_{A}\right) \rightarrow \eta, \vec{x} / a \rightarrow \vec{x}, t / \tau_{A} \rightarrow t$, in which $a$ is the scale length, $\rho_{00}, B_{00}$ are constant, $V_{A}=\sqrt{B_{00}^{2} / \mu_{0} \rho_{00}}$ is the Alfvén speed, and $\tau_{A}=a / V_{A}$ is Alfvénic time. Lundquist number is defined as $\mathrm{S}=V_{A} L / \eta$, where $\mathrm{L}$ is characteristic length of the system. The conservative perturbed MHD[35],[36] is applied in numerical calculations. Each variable, e.g. the plasma density $\rho$, is split into an equilibrium part and a perturbation part of the form $\rho=\rho_{0}+\tilde{\rho}$, where the subscript 0 represents the equilibrium part and the above tilde is for the perturbation. The conservative perturbed resistive MHD model can be expressed in a general form:

$$
\partial_{t} \widetilde{U}+\nabla \cdot(\tilde{\vec{F}}-\widetilde{\bar{D}})=0
$$

where $U=\left(\rho, \rho u_{x}, \rho u_{x}, \rho u_{x}, \rho e, B_{x}, B_{y}, B_{z}\right)^{T}$ are the original conservative variables. $\widetilde{U}=U-U_{0}$ are perturbed conservative variables, $\tilde{\vec{F}}=\vec{F}-\vec{F}_{0}$ is the corresponding perturbed convective flux, and $\widetilde{\vec{D}}=\vec{D}-\vec{D}_{0}$ is the diffusive flux. Such a model is numerically solved by using a flux vector splitting base finite difference method[32]-[34] and has been verified numerically before[35],[36].

The computation is carried out in a two dimensional rectangle domain, $-2 \leq \mathrm{x} \leq 2$ and $-4 \leq$ $\mathrm{y} \leq 4$. Periodical and free boundary condition are imposed at $\mathrm{x}$ and $\mathrm{y}$ direction, respectively. Although all components of magnetic field are evolved in time, it is convenient to define the flux function $\psi$ by $\vec{B}=\nabla \psi \times \vec{e}_{z}+B_{z} \vec{e}_{z}$, so that the constant $\psi$ surfaces are the magnetic surfaces. The equilibrium magnetic field is given by:

$$
B_{x}(y)=B_{x 0}\left(\tanh \frac{y-y_{0}}{d}-\tanh \frac{y+y_{0}}{d}+1\right), B_{y}=0, B_{z}=B_{z 0},
$$

the centers of the two equilibrium current sheets are located at $\pm y_{0}$, and $d$ is half thickness of each current sheet. The equilibrium flow is assumed to be zero, and the plasma pressure is determined by the force balance condition

$$
p_{0}+\frac{1}{2} B_{0}^{2}=(\beta+1) \cdot \frac{1}{2} B_{\infty}^{2},
$$

where $\beta$ is a parameter and $B_{\infty}$ is asymptotic magnetic field for very large $y$. A constant temperature $T_{0}$ for the equilibrium is assumed, and the equilibrium plasma density profile is obtained from $\rho_{0}=p_{0} / T_{0}$. A numerical scheme with 5 th order accuracy in space is applied, which has a much better resolution than that with a lower orders. Usually $501 \times 1001$ grids are used in calculations, and unless otherwise stated, $d=0.2, B_{z 0}=1, \beta=0.2, v=1 \times 10^{-5}, \mathrm{~S}=$ $1 \times 10^{5}$ and $T_{0}=0.1$. The equilibrium parameters are symmetric about both $\mathrm{x}$ and $\mathrm{y}$ axis. 
The initially perturbation in the magnetic flux function is of the form

$$
\delta \psi=\varepsilon\left[\exp \left(-\frac{\left(y-y_{0}\right)^{2}}{d^{2}}\right)+\exp \left(-\frac{\left(y+y_{0}\right)^{2}}{d^{2}}\right)\right] \cdot \cos \left(\frac{\pi}{2} x\right),
$$

where the perturbation amplitude $\varepsilon$ is set to $10^{-3}$. The linear mode growth gate for such type of perturbation (with mode number $\mathrm{k}=1$ ) is significantly larger than that with $\mathrm{k} \geq 2$. When starting from small amplitudes of initial perturbations in calculations, the $\mathrm{k}=1$ perturbation dominates the linear and earlier nonlinear mode growth due to its larger growth rate. The initial perturbation of the magnetic flux is symmetric about y axis. It can be found from Eqs. (1) - (4) that the time evolution of the system does not change the symmetry, so that this symmetry should be maintained during the nonlinear mode evolution.

\section{Numerical results}

It was reported in a recent work[29] that with a low guiding field $B_{z 0}$, small $\mathrm{y}_{0}$ and resistivity $\eta$, long and thin current sheet can be formed during the fast growing phase of kinetic energy, and the secondary islands can form. In a recent work by Nemati et al[30], it was also observed that the dynamics in nonlinear evolution of DTM with higher Lundquist number, $\mathrm{S}=1 \times 10^{5}$, are qualitatively different from that with lower ones. The kinetic energy can reach a higher level during the fast reconnection phase, and it is explained in terms of the secondary tearing instability on the DTM reconnection current ribbons and the generation of small plasmoids. However, the symmetry is eventually lost in the simulation of these studies, so that these tertiary islands are moved asymmetrically out of the reconnection region, coalesce with open field lines and then disappear[30]. In our simulations, the symmetry of magnetic configuration is preserved, allowing us to study the nonlinear dynamics and saturation of DTM under this condition.

\subsection{Effect of Lundquist number}

Numerical scans have been carried out in our calculations to study the effect of the Lundquist number, indicating that it plays an essential role for the onset of plasmoids, as found before[30],[31]. With $y_{0}=0.45$, no plasmoids formation is found in our numerical calculations for $\mathrm{S}=5 \times 10^{3}$ or lower values. When using $S=1 \times 10^{4}$, one secondary island is formed during the nonlinear mode growth, since the current sheet around the $\mathrm{x}$-point of the main island becomes tearing unstable, as shown Figs.1 (a) and (b). The secondary island grows in size while the main island is squeezed (Fig.1 (c) at $\mathrm{t}=116$ ). The system finally decays to parallel field lines (Fig.1 (d) at $\mathrm{t}=168$ ).

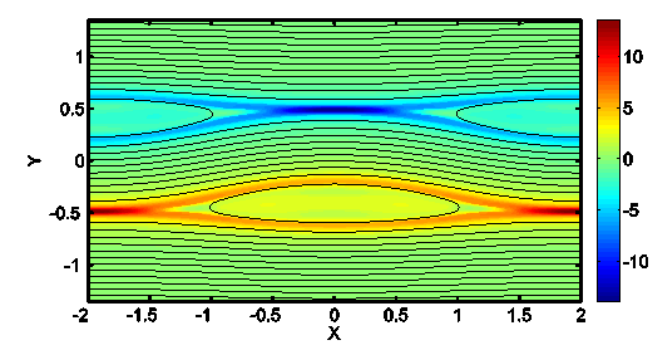

(a) $t=60$

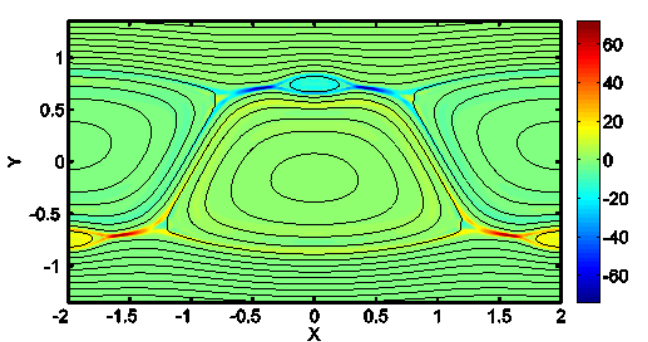

(b) $t=96$ 


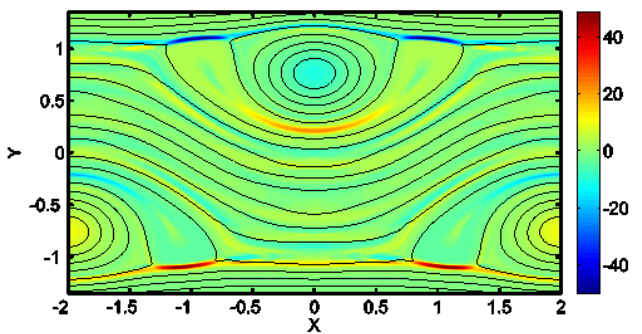

(c) $t=116$

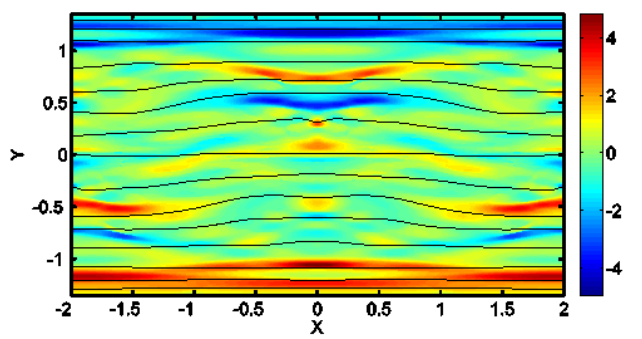

(d) $t=168$

Figure 1 Evolution of magnetic configuration and current density distribution $J_{z}$ with $\mathrm{S}=1 \times 10^{4}$ and $y_{0}=$ 0.45 at (a) $t=60$, (b) $t=96$, (c) $t=116$, (d) $t=168$.

For an even higher $\mathrm{S}$ value, $\mathrm{S}=1 \times 10^{5}$ (with $y_{0}=0.45$ ), the time evolution of kinetic energy $\mathrm{E}_{\mathrm{K}}$ is shown in Fig. 2 in blue solid line, and the change of magnetic energy $\left|\delta E_{M}\right|, \delta \mathrm{E}_{\mathrm{M}}=\mathrm{E}_{\mathrm{M}}(t)-$ $\mathrm{E}_{\mathrm{M}}(t=0)$ in black dashed line. After an initial phase and a slow Rutherford phase, the kinetic energy starts to grow quickly at $t=135$. However around $t=157$ it falls down a little bit and goes up again until it reaches the maximum value around $t=173$. The perturbed magnetic energy $\left|\delta \mathrm{E}_{\mathrm{M}}\right|$, experiences a fast increase between $\mathrm{t}=145$ and $\mathrm{t}=175$, indicating the change of the magnetic configuration. Then with small amplitude oscillation, $\left|\delta \mathrm{E}_{\mathrm{M}}\right|$ enters a very long and slow varying phase.

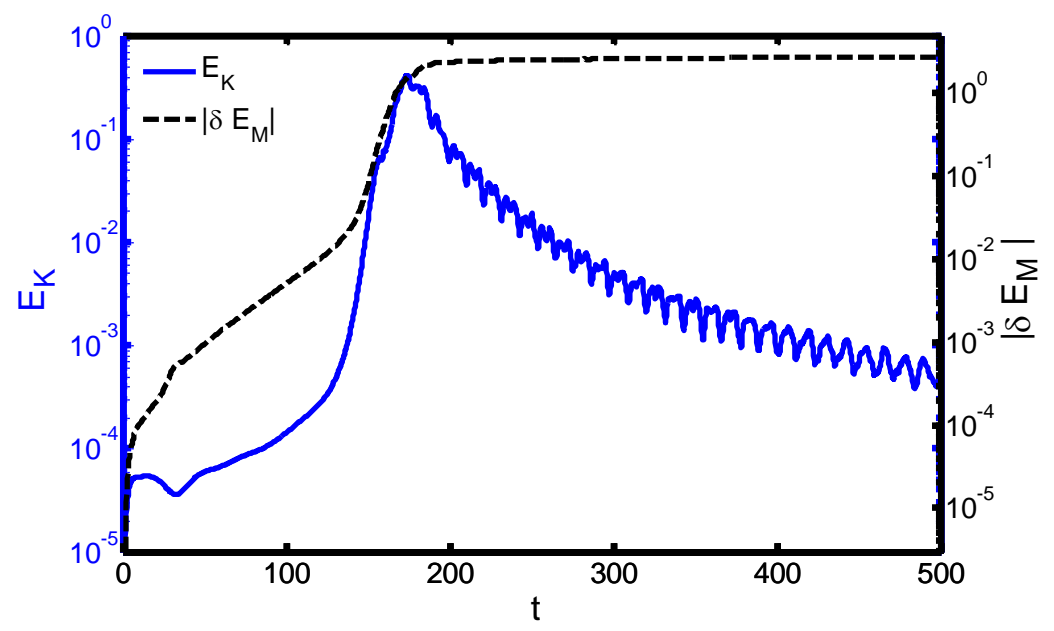

Figure 2 Time evolution of kinetic energy $E_{K}$ and the change of magnetic energy $\left|\delta E_{M}\right|$ for $S=1 \times 10^{5}$ and $y_{0}=0.45$. The solid curve is for $\mathrm{E}_{\mathrm{K}}$ and the dashed line is for $\left|\delta \mathrm{E}_{\mathrm{M}}\right|$.

Corresponding to Fig.2, in Fig.3 the contour lines of magnetic flux function and the color map of current density $\mathrm{J}_{\mathrm{z}}$ along $\mathrm{z}$ direction are shown at different time. With the nonlinear growth of magnetic islands on each original equilibrium current sheet, a new elongated reconnection region with high current density is formed, as shown at $\mathrm{t}=158$ in Fig.3(a). The maximum current density is $\left|J_{z}\right|_{\max }=$ 67.9. This newly formed current sheet becomes tearing unstable and small plasmoids are generated symmetrically. These small islands are ejected out and vanish repeatedly until a secondary island starts to grow up at the center of the current sheet and divide it into two current ribbons, as indicated in Fig.3(b) at $\mathrm{t}=161$. This secondary island keeps growing up in size and squeezes the up-moving main island into a newly formed thin curved current sheet, as shown in Fig.3(c), (d) and (e) at t=167, t=173, $\mathrm{t}=180$, respectively. Once again, this curved current sheet becomes unstable, and tertiary islands onsets at its center (Fig.3(f) at $\mathrm{t}=182$ ). Since symmetry is well preserved in our simulation, this tertiary island 
does not escape from the central region to positive or negative $\mathrm{x}$ direction. And it grows bigger while the secondary island shrinks until these two islands become comparable in size at $\mathrm{t}=195$, as seen from Fig.3(g). Then the system enters into a quasi-stationary state characterized as two pairs of coexisting islands which only changes slowly at global current diffusion time. A typical picture of such quasi-stationary state is shown in Fig.3(h) at $\mathrm{t}=400$. It is worth to point out this quasi-stationary state is achieved only when the symmetry is well preserved in numerical calculations.

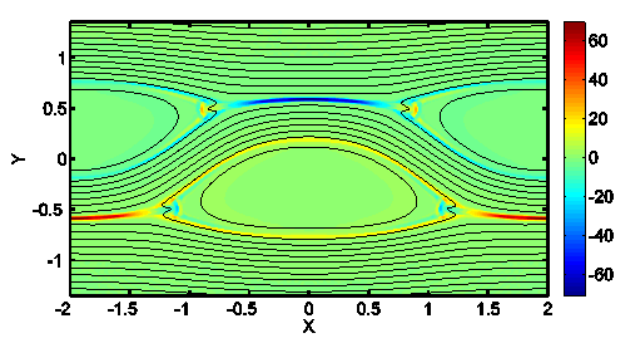

(a) $t=158$

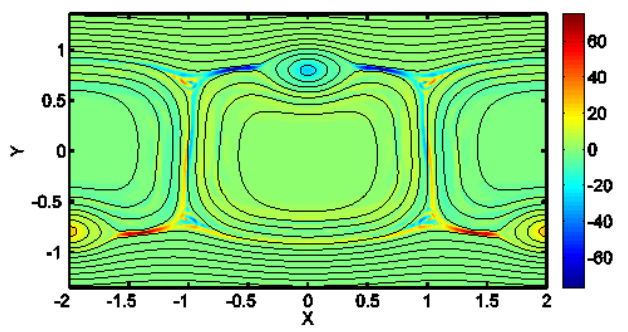

(c) $\mathrm{t}=167$

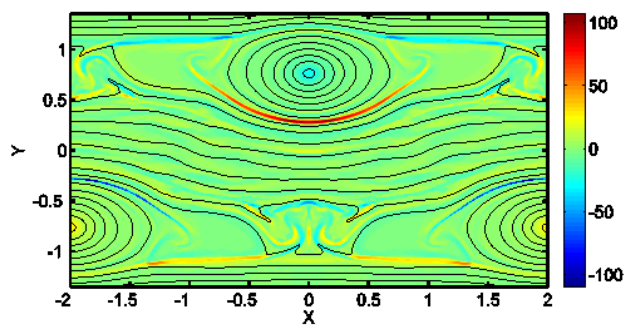

(e) $t=180$

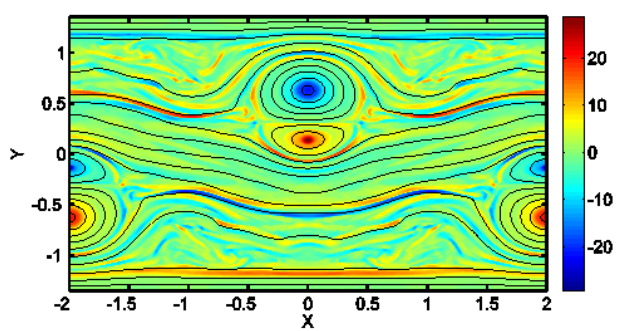

(g) $t=195$

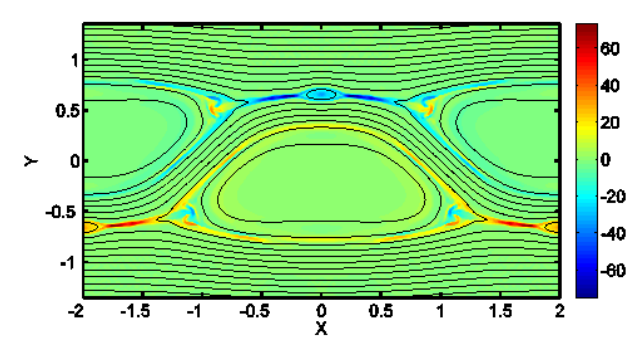

(b) $t=161$

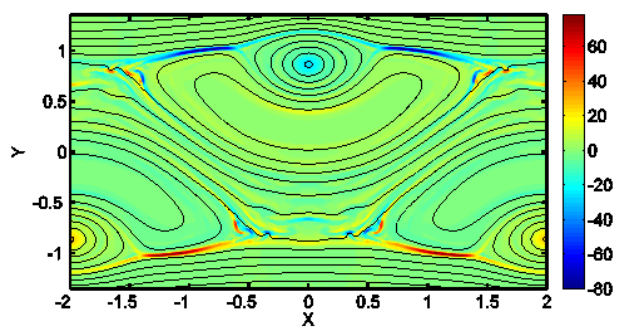

(d) $t=173$

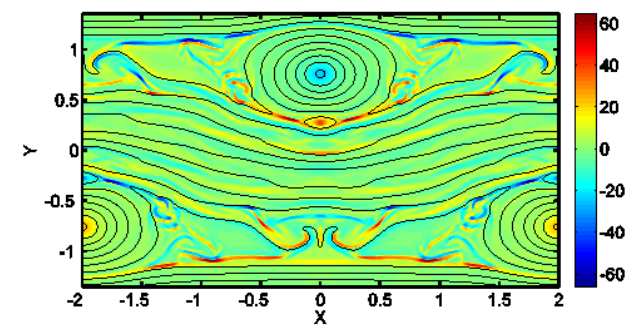

(f) $t=182$

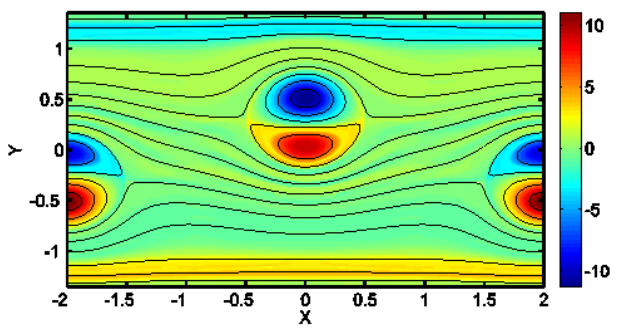

(h) $t=400$

Figure 3 Evolution of magnetic configuration and current density distribution $J_{z}$ with $\mathrm{S}=1 \times 10^{5}$ and $y_{0}=$ 0.45 at (a) $t=158$, (b) $t=161$, (c) $t=167$, (d) $t=173$, (e) $t=180$, (f) $t=182$, (g) $t=195$, (h) $t=400$.

Similar plasmoids formation and final quasi-stationary state have been reported during the nonlinear evolution of resistive internal kink[38],[39] for a sufficiently high S number. Secondary and tertiary islands are also observed to onset during the fast reconnection, and eventually the system 
reaches a helical equilibrium with two coexisting magnetic islands. There is a similar basic physical

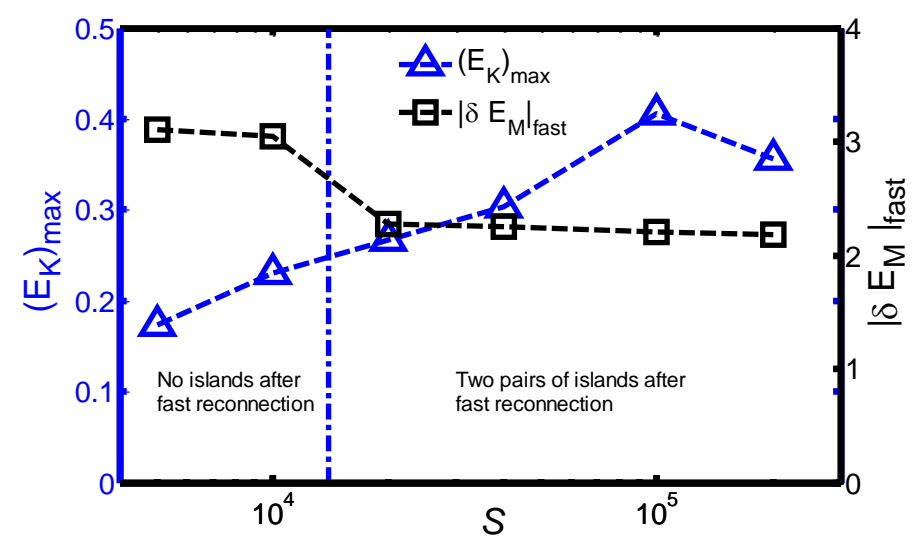

Figure 4 The maximum kinetic energy $\left(E_{K}\right)_{\max }$ and magnetic energy released after fast reconnection $\left|\delta E_{M}\right|_{\text {fast }}$ as a function of Lundquist number $S$ with $y_{0}=0.45$. The dash dot line schematically separates the two kinds of evolutions. The points on the left stand for the evolutions that finally decay into almost parallel field lines, and the points on the right stand for the evolution that saturate with two pairs of islands.

process for both cases, the formation of thin current sheet and subsequent plasmoids during the nonlinear mode evolution. In the simulation of resistive internal kink, the conservation of symmetry is essential, as also found in our simulations.

It is recognized[30] that due to qualitatively different dynamic with a higher $\mathrm{S}$, the maximum kinetic energy can reach a higher level. The maximum kinetic energy and magnetic energy released during fast reconnection is also investigated here for different $\mathrm{S}$ values. Fig.4 displays $\left(E_{K}\right)_{\max }$ and $\left|\delta E_{M}\right|_{\text {fast }}$ as function of $\mathrm{S}$, where $\left(E_{K}\right)_{\max }$ is maximum kinetic energy, and $\left|\delta E_{M}\right|_{\text {fast }}$ is magnetic energy released just after the fast reconnection. For the cases with $S \leq 1 \times 10^{4}$, the final magnetic configurations are almost parallel field lines. For other cases with higher $\mathrm{S}$ values, however, after fast reconnection the system saturates at quasi-stationary state with two pairs of islands, thus less magnetic energy is released. $\left(E_{K}\right)_{\max }$ increases with increasing $\mathrm{S}$ except for $\mathrm{S} \geq 1 \times 10^{5}$, being different from that of Ref. [30].

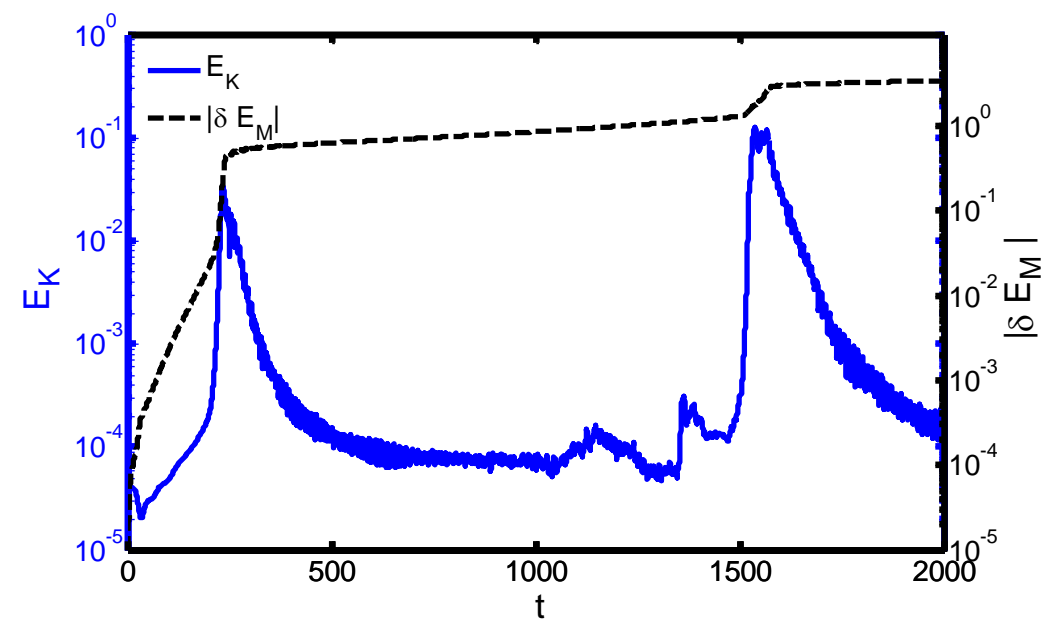

Figure 5 Evolution of kinetic energy $\mathrm{E}_{\mathrm{K}}$ and the absolute variation of magnetic energy $\left|\delta \mathrm{E}_{\mathrm{M}}\right|$ for $S=1 \times$ $10^{5}$ and $y_{0}=0.6$. 


\subsection{Effect of the distance between current sheets}

In addition to the $S$ value, the half distance between the equilibrium current sheets, $y_{0}$, also significantly affects the plasmoids formation during nonlinear DTM evolution. Fixing the value of $S=$ $1 \times 10^{5}$, for a small $y_{0}=0.15$ no plasmoids formation is observed, and the fast reconnection is absent for even smaller $y_{0}$ due to the reduction of free energy. This is qualitatively consistent with Ref[29]. For $y_{0}=0.3$, only one secondary island forms, but there's no tertiary islands and no final quasi-stationary state with two co-existing islands. With a larger half distance $y_{0}=0.6$, a new evolution process with two fast reconnection phases is found. Fig.5 gives the evolution of kinetic energy $E_{K}$ in blue solid line and the absolute variation of magnetic energy $\left|\delta E_{M}\right|$ in black dashed line in this case. There are two fast evolving phases for both kinetic and magnetic energy. The kinetic energy reaches its first maxima at $\mathrm{t}=234$ and then decays. $\left|\delta E_{M}\right|$ starts to increase quickly at $\mathrm{t}=217$, which is a little later than the kinetic energy, and ends up at $t=248$. After quite a long time period, the kinetic energy enters the second fast growing phase at $\mathrm{t}=1504$, reaching the second maxima at $\mathrm{t}=1537$. The second fast magnetic energy decrease starts at $t=1511$ and ends up at about $t=1585$. During the second fast reconnection, more than three times magnetic energy is released compared to the first one, and kinetic energy reaches a higher level.

Corresponding to Fig.5, Fig.6 shows the evolution of magnetic field and current density. In the island's x-point region, the elongated current sheet is formed and grows in length and amplitude, as shown in Fig.6(a). A secondary island then births up and grows at the center of the upper current sheet, as indicated in Fig.6(b) at $\mathrm{t}=238$, dividing the current sheet into two current ribbons. The secondary island continues to grow until its two sides touch the edges of the upper main island. At the same time current ribbons become shorter and weaker, with the maximum current density $\left|J_{z}\right|_{\max }=14.7$. The disappearance of the current ribbons terminates the first quick decreasing phase of magnetic energy (Fig.6(c) at $\mathrm{t}=250$ ). Before the second fast reconnection, the system experiences a long and slow varying stage, lasting more than 1000 time period, as seen from Fig.6(d). The structure of the magnetic configuration changes a little, i.e., the main island expands along y direction in this period of time. As a result of this small expansion, the current ribbons with high current density $\left|J_{z}\right|_{\max }=70.9$ is recovered, triggering the second fast reconnection phase, and the central main island is squeezed, as illustrated in Fig.6(e) at $\mathrm{t}=1540$. The consequent magnetic configuration shown in Fig.6(f) at $\mathrm{t}=2000$ is similar to the quasi-stationary state with two pairs of islands shown in Fig. 3(h) for the $y_{0}=0.45$ case.

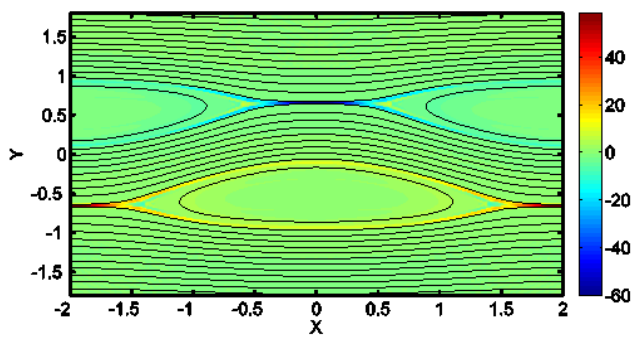

(a) $t=234$

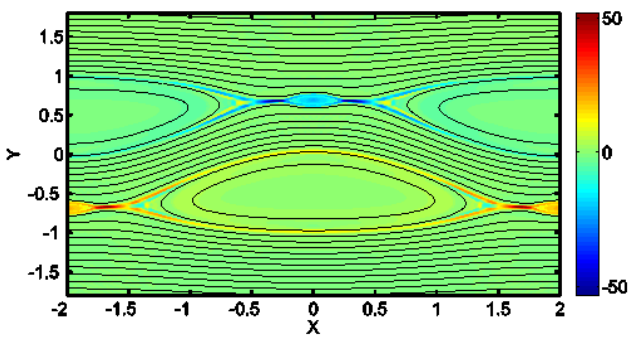

(b) $t=238$ 


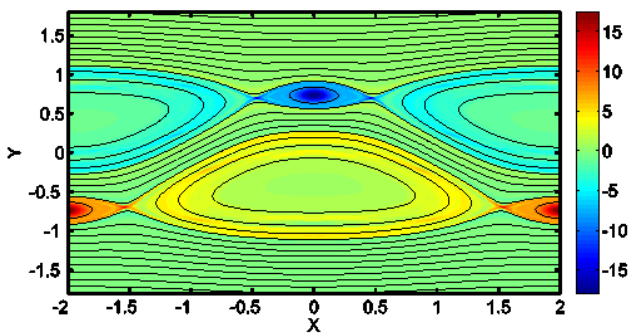

(c) $t=250$

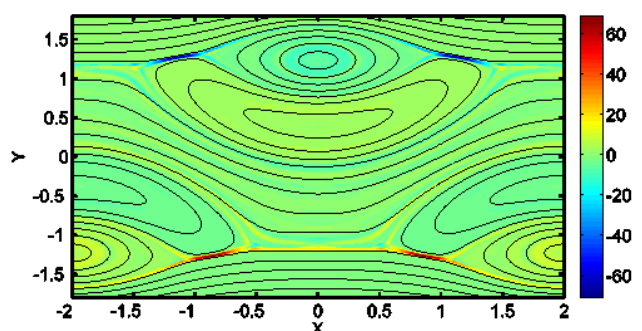

(e) $\mathrm{t}=1540$

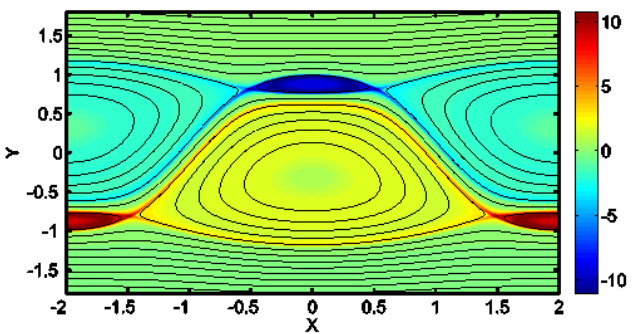

(d) $\mathrm{t}=1470$

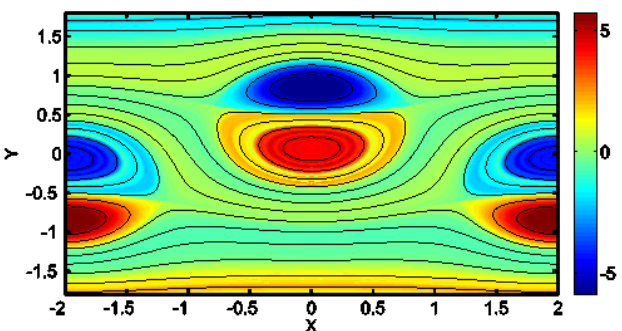

(f) $\mathrm{t}=2000$

Figure 6 Evolution of magnetic configuration and current density distribution $J_{z}$ with $S=1 \times 10^{5}$ and $y_{0}=0.6$ at (a) $\mathrm{t}=234$, (b) $\mathrm{t}=238$, (c) $\mathrm{t}=250$, (d) $\mathrm{t}=1470$, (e) $\mathrm{t}=1540$, (f) $\mathrm{t}=2000$.

For the above high $\mathrm{S}$ simulations, the thin and high density current sheets and ribbons dominate the fast reconnection, driving the flows for main islands deformation which in turn further stretch high current density regions. The second fast reconnection phase is mainly due to the competition between elongating and shortening effects upon current ribbons, induced by the deformation of main islands and enlargement of secondary islands, respectively. The triangular deformation of main islands tends to stretch the current ribbons along $\mathrm{x}$ direction, while the growth of secondary islands tends to annihilate them. Being different from the $y_{0}=0.45$ case, the current ribbons for $y_{0}=0.6$ case are shorter and are "swallowed" by expansion of secondary islands before enough deformation of main islands. This halts the first fast reconnection phase. As the current ribbons shrink to much weaker current points, the system enters a long and slow current diffusion phase until the deformation of main islands is large enough to create the current ribbons once again. This leads to the second fast reconnection phase. Symmetry is also necessary for the halt of fast reconnection. With a small asymmetry the secondary island will be ejected out to either left or right side, and it will coalesce with the main island, so that there will be no second fast reconnection phase.

When further increasing $y_{0}$ to 0.9 , a fast reconnection phase is found to be halted by the arising of a secondary island (Fig.7 (a) at $\mathrm{t}=390$ ). However, the main islands interact weakly with each other, and the secondary island slowly shrinks (Fig.7 (b) at $\mathrm{t}=1000$ ) to a X point (Fig.7 (c) at $\mathrm{t}=1500$ ). The system then slowly saturates without the further variation of the magnetic configurations (Fig.7 (d) at $\mathrm{t}=2500)$. 


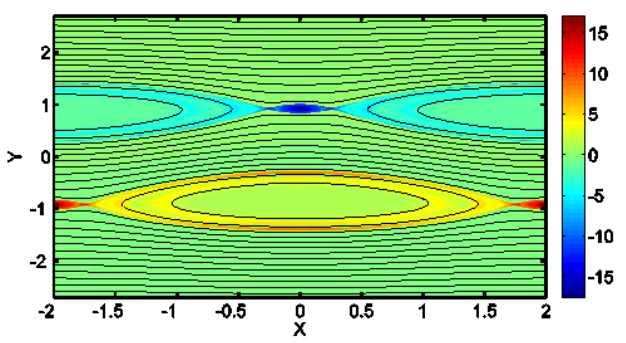

(a) $t=390$

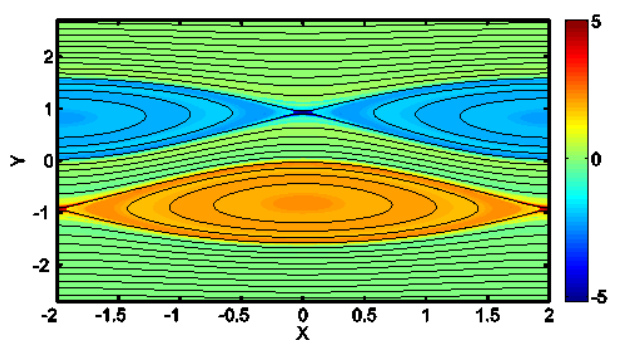

(a) $\mathrm{t}=1500$

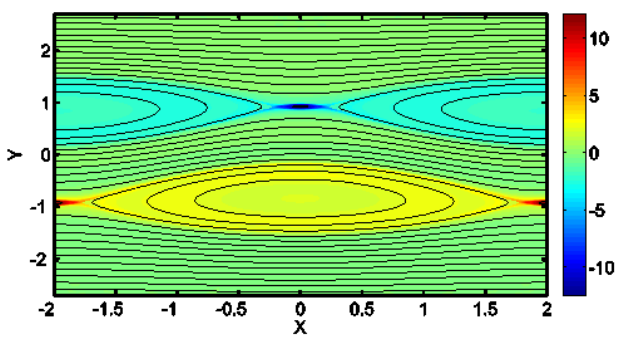

(b) $t=1000$

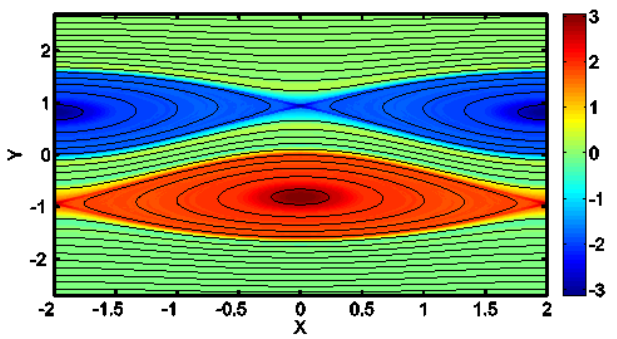

(b) $t=2500$

Figure 7 Evolution of magnetic configuration and current density distribution $J_{z}$ with $S=1 \times 10^{5}$ and $y_{0}=$ 0.9 at (a) $\mathrm{t}=390$, (b) $\mathrm{t}=1000$, (c) $\mathrm{t}=1500$, (d) $\mathrm{t}=2500$.

Finally, the nonlinear behavior of DTM is shown in the $\left(\mathrm{S}, y_{0}\right)$ plane. In general, the Lundquist number $S$ plays an essential role for plasmoids generation. For higher $S$, the current sheets can become more unstable and easier to break up. Secondary islands are common for $S \geq 10^{4}$, and the generation of tertiary islands or an evolution including two fast reconnections require even higher $S$. On the other hand, plasmoids generations take place with a moderate $y_{0}$. For a small $y_{0}$, the free energy is limited so that no plasmoids are observed; while for a too large $y_{0}$, the fast reconnection is halted due to the weak interaction between the main islands, and they saturated in the conventional way like the single tearing mode.

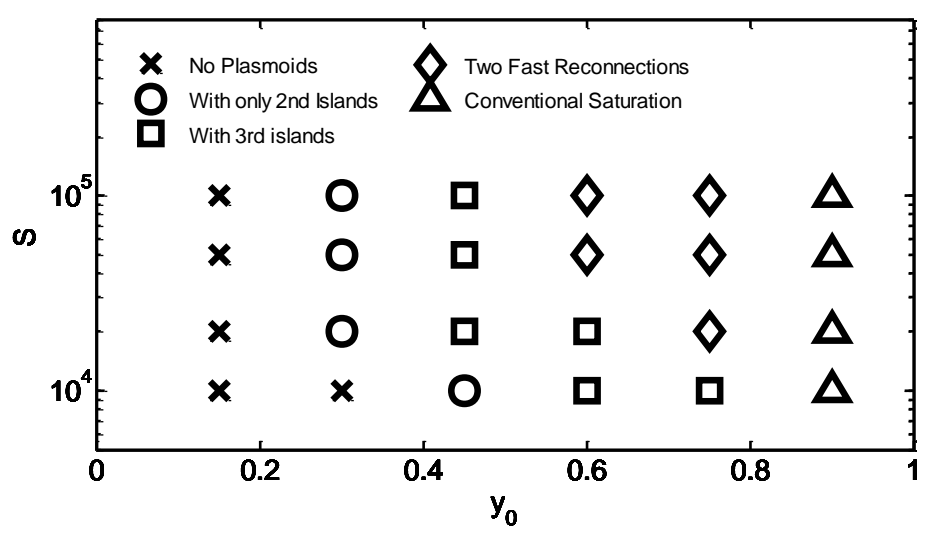

Figure 8 Behaviors of nonlinear DTM in the $\left(S, y_{0}\right)$ plane. Five different types of nonlinear evolution are marked: No plasmoids generation (crosses), with only secondary islands generation (circles), with tertiary islands generation (squares), having two fast reconnections in mode evolution (diamonds), and conventional islands saturation (triangles).

\section{Discussion and summary}


The non-monotonic q-profile is of great interest for the steady operation of a fusion reactor, since it is associated with the formation of the internal transport barrier and a higher bootstrap current fraction than the standard H-mode plasmas[6],[7]. However, such configuration can be unstable to the DTM, which can destroy the internal transport barrier and cause confinement degradation[20]-[23]. Understanding of the nonlinear growth and saturation of DTM remains to be an important issue for fusion plasmas. It was well known from previous numerical studies with relatively low Lundquist number that the key dynamics of nonlinear DTM is the formation of intensive current points and explosive magnetic reconnection in the nonlinear phase. The Lundquist number can reach $10^{6}$ or higher in existing tokamak plasmas. Recent studies with the Lundquist number being closer to that of existing tokamak plasmas revealed the formation of the secondary islands[29] or tertiary islands[30][31] during the nonlinear DTM growth. However, in these studies the symmetry is not preserved in calculations, so that the plasmoids only survive transiently in the reconnection region in the nonlinear phase caused by numerical errors, corresponding to an artificial generation of plasma flow and the loss of momentum conservation. The conservative perturbed resistive MHD model[35],[36] allows the symmetry well preserved during the nonlinear mode growth and saturation in numerical simulations. Utilizing this model, our numerical calculations show that for sufficiently high Lundquist number, the nonlinear evolution of DTM is significantly affected by plasmoids. The system can saturates at a state with secondary and tertiary islands. The distance between the two resonant surfaces is also found to be important in determining the nonlinear mode growth, since it affects the evolution of the secondary island. These results indicate the important role of plasmoids in determining the nonlinear dynamics of DTM, which is quite different from the conventional understanding basing on the simulation results with lower Lundquist number or without keeping the symmetry in calculations.

In summary, the nonlinear evolution of double tearing mode is investigated numerically with conservative perturbed resistive MHD model. The nonlinear dynamics in low and high Lundquist number regimes are found to be quite different. For a low $S$ value $\left(\sim 10^{3}\right)$, no plasmoids are observed in calculations, as expected. With $\mathrm{S} \sim 10^{5}$, secondary and tertiary islands are found to be generated, resulting in the system to saturate at a quasi-stationary state with these islands coexisting in two pairs. The distance between the two resonant surfaces is also important in determining the reconnection process. For a small distance between the two resonant surfaces, $y_{0} \leq 0.15$, plasmoids are not observed in numerical calculations. For a sufficiently large distance between the two resonant surfaces, $y_{0}=0.9$, one fast reconnection phase exists, generating the secondary island which decays later, and finally the main islands saturate. For an intermediate distance of equilibrium current sheets, plasmoids formation dominates the nonlinear mode growth and saturation. Moreover, a new kind of nonlinear evolution with two fast reconnection phases is found due to the evolution of current ribbons of the secondary island. Symmetry in our simulation is essential for obtaining both the second fast reconnection and the final quasi-stationary magnetic configuration with two pairs of islands. Our results indicate that the nonlinear growth and saturation of DTM are affected by the formation and evolution of plasmoids, which are determined by the distance between the two resonant surfaces and the Lundquist number. These results reveal that the nonlinear DTM growth in high Lundquist number regime, being close to that of existing tokamak plasmas, can be quite different from that with low Lundquist numbers. 


\section{ACKNOWLEGDEMENTS}

The numerical calculations are performed on ShenMa High Performance Computing Cluster at the Institute of Plasma Physics, Chinese Academy of Sciences. This work is supported by the National Natural Science Foundation of China under Grant No. 11475219, the Science Foundation of the Institute of Plasma Physics, Chinese Academy of Sciences (DSJJ-15-JC02), and the National Magnetic Confinement Fusion Science Program of China under Grant No. 2013 GB111002.

\section{References}

[1] N. F. Loureiro and D. A. Uzdensky, Plasma Phys. Control. Fusion 58, 014021(2016).

[2] N. F. Loureiro, A. A. Schekochihin, and S. C. Cowley, Phys. Plasmas 14, 100703(2007).

[3] N. F. Loureiro, A. A. Schekochihin, and D. A. Uzdensky, Phys. Rev. E 87, 013102(2013).

[4] R. Samtaney, N. F. Loureiro, D. A. Uzdensky, A. A. Schekochihin, and S. C. Cowley, Phys. Rev. Lett. 103, 105004(2009).

[5] L. Ni, K. Germaschewski, Y. Huang, B. P. Sullivan, H. Yang, and A. Bhattacharjee, Phys. Plasmas 17, 052109(2010).

[6] L.-G. Eriksson, et al, Phys. Rev. Lett. 88, 145001(2002).

[7] E. Joffrin, et al, Nucl. Fusion 43, 1167(2003).

[8] V. S. Titov, K. Galsgaard, and T. Neukirch, Astrophys. J. 582, 1172 (2003).

[9] K. Kusano,T. Maeshiro, T. Yokoyama, and T. Sakurai, Astrophys. J. 610, 537 (2004).

[10] D. W. Longcope, D. E. McKenzie, J. Cirtain, and J. Scott, Astrophys. J. 630, 596 (2005).

[11] K. Bowers and H. Li, Phys. Rev. Lett. 98, 035002 (2007).

[12] Y. Koide, M. Kikuchi, M. Mori, S. Tsuji, S. Ishida, N. Asakura, Y. Kamada, T. Nishitani, Y. Kawano, T. Hatae, T. Fujita, T. Fukuda, A. Sakasai, T. Kondoh, R. Yoshino, and Y. Neyatani, Phys. Rev. Lett. 72, 3662 (1994).

[13] L. L. Lao, K. H. Burrell, T. S. Casper, V. S. Chan, M. S. Chu, J. C. DeBoo,E. J. Doyle, R. D. Durst, C. B. Forest, C. M. Greenfield, R. J. Groebner, F.L. Hinton, Y. Kawano, E. A. Lazarus, Y. R. LinLiu, M. E. Mauel, W. H.Meyer, R. L. Miller, G. A. Navratil, T. H. Osborne, Q. Peng, C. L. Rettig,G. Rewoldt, T. L. Rhodes, B. W. Rice, D. P. Schissel, B. W. Stallard, E. J.Strait, W. M. Tang, T. S. Taylor, A. D. Turnbull, R. E. Waltz, Phys. Plasmas 3, 1951 (1996).

[14] Q. Yu and S. Günter, Nucl. Fusion 39, 487 (1999).

[15] P. L. Pritchlett, Y. C. Lee and J. F. Drake, Phys. Fluids 23, 1368(1980).

[16] E. Fredrickson, R. V. Budny, D. Darrow, G. Y. Fu, J. Hosea, C. K. Phillips, J. R. Wilson and J. W. Van Dam, Phys. Plasmas 7, 4112 (2000).

[17] M. Janvier, Y. Kishimoto, and J. Q. Li, Phys. Rev. Lett. 107, 195001 (2011).

[18] J. Q. Dong. M. Mahajan, and W. Horton, Phys. Plasmas 10, 3151 (2003).

[19] J. Q. Dong, Z. Z. Mou, Y. X. Long and S. M. Mahajan, Phys. Plasmas 11, 5673 (2004).

[20] Z. Chang,W. Park, E. D. Fredrickson, S. H. Batha, M. G. Bell, R. Bell, R.V. Budny, C. E. Bush, A. Janos, F. M. Levinton, K. M. McGuire, H. Park,S. A. Sabbagh, G. L. Schmidt, S. D. Scott, E. J. Synakowski, H. Takahashi,G. Taylor, M. C. Zarnstorff, Phys. Rev. Lett. 77, 3553 (1996).

[21] M. R. de Baar, et al, Phys. Rev. Lett. 78, 4573 (1997).

[22] M Zabiégo, et al, Plasma Phys. Control. Fusion 43, 1625(2001). 
[23] P. Maget, et al, Phys. Plasmas 14, 052509(2007).

[24] Y. Ishii, M. Azumi, G. Kurita, and T. Tuda, Phys. Plasmas 7, 4477 (2000).

[25] Y. Ishii, M. Azumi, and Y. Kishimoto, Phys. Rev. Lett. 89, 205002 (2002).

[26] Y. Ishii, M. Azumi, Y. Kishimoto, and J. N. Leboeuf, Nucl. Fusion 43,539 (2003).

[27] Z. X. Wang, X. G. Wang, J. Q. Dong, Y. A. Lei, Y. X. Long, Z. Z. Mou, and W. X. Qu, Phys. Rev. Lett.99, 185004(2007).

[28] Z. X. Wang, X. G. Wang, J. Q. Dong, Y. Kishimoto, and J. Q. Li, Phys. Plasmas15, 082109(2008).

[29] C. L. Zhang, Z. W. Ma, Phys. Plasmas 18, 052303(2011).

[30] M. J. Nemati, Z. X. Wang, and Lai Wei, The Astrophysical Journal, 821, 128(2016).

[31] M. J. Nemati, Z. X. Wang, and Lai Wei, The Astrophysical Journal, 835, 191(2017).

[32] C. W. Shu, ICASE Report No. 97-65(1997).

[33] A. K. Henrick, T. D. Aslam, J. M. Powers, J. Comput. Phys. 207, 542(2005).

[34] C. W. Shu, J. Comput. Phys.316, 598(2016).

[35] J. Ma, W. Guo, Z. Yu, Commun. Comput. Phys. 21, 1429(2017).

[36] W. Guo, J. Ma, Z. Yu, Phys. Plasmas 24, 032115(2017).

[37] J. P. Goedbloed, S. Poedts, Principles of Magnetohydrodynamics with Applications to Laboratory and Astrophysical Plasmas (Cambridge University Press, New York, 2004).

[38] Q. Yu, S. Gütner, and K. Lackner, Nucl. Fusion 54, 072005(2014).

[39] S. Gütner, Q. Yu, K. Lackner, A. Bhattacharjee, and Y-M Huang, Plasma Phys .Control. Fusion 57, 014017(2014). 